\title{
Internet-Based Heat Evaluation and Assessment Tool (I-HEAT): Feasibility Analysis of a Visualization and Decision-support Tool for Extreme Heat Preparedness in Detroit, Michigan
}

\author{
NATALIE R. SAMPSON ${ }^{1 *}$, KATHRYN C. CONLON ${ }^{2}$, ROBERT ROMMEL ${ }^{3}$, \\ GEOFFREY JACQUEZ ${ }^{3,4}$, MARIE S. O'NEILL ${ }^{5}$ \\ Volume 2, Fall 2014 \\ http://dx.doi.org/10.3998/mjs.12333712.0002.006 \\ 1'Department of Health and Human Services, University of Michigan-Dearborn, \\ 19000 Hubbard Dr., Dearborn, Ml 48126 \\ ${ }^{2}$ National Center for Atmospheric Research, P.O. Box 3000, Boulder CO 80307 \\ ${ }^{3}$ BioMedware, 121 West Washington, Ann Arbor, MI 48104 \\ ${ }^{4}$ Department of Geography, 112 Wilkeson Hall, \\ State University of New York at Buffalo, Buffalo, New York 14261 \\ ${ }^{5}$ Departments of Environmental Health Sciences and Epidemiology, \\ University of Michigan School of Public Health, 1415 Washington Heights, \\ Ann Arbor, MI 48109 \\ *Corresponding author: Natalie R. Sampson, nsampson@umich.edu
}

\section{ABSTRACT}

Observed and expected changes in global and regional climates pose significant challenges to local decision makers tasked with identifying, preparing for, and responding to impacts on human populations. In particular, extreme heat is expected to increase in duration and intensity in Midwestern U.S. cities in this century. Because of the increased availability of climatic and demographic data at fine spatial scales (regional and neighborhood levels, respectively) (Walsh et al. 2014; Bhaduri et al. 2007), data-driven applications for identification of intra-urban vulnerability to temperature and climate are becoming possible. As one example, the InternetBased Heat Evaluation and Assessment Tool (I-HEAT) integrates multi-scale re- 
motely sensed environmental imagery with demographic and health data for Detroit, Michigan in a web-based mapping software. Few published feasibility studies document how these tools are made usable for and evaluated by the end-users leading heat preparedness efforts in the U.S., such as emergency managers, public health practitioners, and sustainability coordinators. In this paper, we describe our process for presenting I-HEAT to a diverse group of 28 end-users in Southeast Michigan during a day-long workshop, where we collected feedback through surveys and focus groups on the tool's real-world application and utility. We present the findings of these surveys and focus groups, highlighting the strengths and limitations of the tool and the additional innovative applications identified by workshop participants. By sharing the process and findings of our feasibility study, we inform future development, implementation, and evaluation for I-HEAT and similar tools that may be critical in assisting local decision makers in their sustainability and climate planning efforts.

\section{Introduction}

\subsection{Climate Change, Extreme Heat, Sustainable Living, and Human Health}

Climate change will increase the duration, frequency, and intensity of heat waves (IPCC 2007), with portions of the United States (U.S.) experiencing more intense and frequent periods of extreme heat than in previous years (Meehl and Tebaldi 2004). The threat of extreme heat to human health is a real and pressing concern. Despite significant protective technological advances (e.g., forecasting meteorological events, increased access to air conditioning) to reduce exposure to high temperatures, extreme heat remains the leading cause of death related to natural disasters in the U.S. (Borden and Cutter 2008). Due to projected climate change, current and future populations face challenges in maintaining the dynamic relationship between natural systems and human health.

The impacts of extreme heat on human health are often difficult to predict or identify and have been assessed via retrospective epidemiological studies. These studies emphasize that heat-related morbidity and mortality depend on the characteristics of the heat event, as well as the health status and geography of the affected population (Basu 2002; Curriero et al. 2002; Anderson and Bell 2011). Physiologically, short-term exposure to increases in ambient temperature (e.g., heat waves) 
can cause the human body to reallocate blood flow from vital organs, such as the heart and lungs, to below the skin's surface in order to thermoregulate. Ineffective thermoregulation, due to aging, medication use, or chronic health conditions (e.g., diabetes), can result in misdirected blood allocation and ultimately contribute to cardiovascular and respiratory stress (Astrand et al. 2003). Population characteristics such as age and health status are commonly used indicators of biological characteristics that contribute to susceptibility to extreme heat.

Climate models suggest that large U.S. urban populations will experience disproportionately extreme heat (Oleson et al. 2013). This is due in part to the urban heat island (UHI) effect where observed ambient temperatures are higher within a city than in the surrounding less-developed, more vegetated suburban and rural areas (Arnfield 2003). Heat is absorbed by cityscape materials like concrete, asphalt and brick, contributing to the UHI effect (Imhoff et al. 2010). U.S. metropolitan centers are projected to grow significantly by the mid to late century; larger and older populations will be living in urban areas (Frumkin 2002) with higher ambient temperatures, potentially leading to a larger-magnitude impact of extreme heat on human health. Some adaptive measures, such as increasing air conditioning prevalence and usage, can limit individual and short-term exposure to extreme heat-but they can also increase ambient temperatures (Sailor and Pavlova 2003) and contribute to global climate change via the release of greenhouse gases and latent heat expenditure (Salamanca et al. 2014). It is critical to consider the net effect of adaptive measures when identifying ways to reduce future risk of extreme heat.

\subsection{Identifying Populations Vulnerable to Heat at the Local Level}

To prevent heat-related morbidity and mortality, local municipalities face an increasing need to identify populations most vulnerable to extreme heat, and spatial analyses using local data may assist these efforts. National research priorities pertaining to climate change and health have garnered attention from public and private entities, and several researchers have called for the integration of climate and health data to better inform climate change mitigation and adaptation planning decisions (Hess, Schramm, and Luber 2014; Bierbaum et al. 2013; Miles et al. 2006). Heat-related vulnerability, as defined by Wilhemi and Hayden (2010), comprises exposure, sensitivity, and adaptive capacity. Scholars and practitioners have mapped this construct in diverse ways, often using a heat vulnerability index (HVI) (Johnson et al. 2012; Reid et al. 2009; Reid et al. 2012; Wolf and McGregor 2013; Loughnan et al. 2013). 
Building on these spatial analyses, organizations that conduct heat preparedness have called for the development of easily accessible tools that can aid in the identification of areas where vulnerable populations reside, as a way to prepare for extreme heat events (O’Neill et al. 2010). Such tools must reach diverse audiences, as heat preparedness efforts are often led by different sectors in different municipalities (White-Newsome et al. 2014), including departments or agencies of aging, sustainability, emergency preparedness, and public health departments. In response, the Internet-based Heat Evaluation and Assessment Tool (I-HEAT) (available at www. biomedware.com/I-Heat/IHeatViewer.html) was recently developed and feasibility tested with diverse stakeholders in Southeast Michigan. I-HEAT is a user-friendly, dynamic mapping tool that concurrently displays HVI data with multi-scale remotely sensed land surface temperatures and measures of vegetation. Users can access I-HEAT online and identify populations at risk from extreme heat in Detroit, Michigan. A series of tabs displays the following information at the census-tract level atop a background map: at-risk areas, satellite and temperature data, demographic data, and HVI values. For further details on the tool and its development, see Conlon et al. (2014).

\subsection{Study Purpose and Objectives}

City and community leaders must prepare locally for projected climate change, including heat-related threats to health. To this end, scholars and practitioners have mapped heat vulnerability using varied data and scales, and some are beginning to develop decision-making application tools based on these analyses. I-HEAT, a web-based interactive mapping software, is one example. There are few reports of feasibility studies that document how these tools are made usable for and evaluated by end-users, such as emergency managers, public health practitioners, and sustainability coordinators. In this paper, we describe our process for presenting I-HEAT to a diverse group of 28 end users in Southeast Michigan during a day-long workshop, Heat and Health in Michigan: An Interactive Workshop on the Development of Risk Assessment Resources, where we facilitated interactions with the tool and collected feedback through surveys and focus groups. We present the findings of this I-HEAT feasibility study and future directions for development, implementation, and evaluation for I-HEAT and similar tools. 


\section{Materials and Methods}

\subsection{Climate Change in Detroit, Michigan}

Michigan has many climate change adaptation efforts underway. The Michigan Department of Community Health (MDCH) participates in the Climate-Ready States and Cities Initiative of the U.S. Centers for Disease Control and Prevention (CDC). Through this program, MDCH utilizes CDC's technical and organizational support in developing Michigan-specific climate change adaptation efforts. As part of the collaboration, MDCH works closely with local governments and officials, including representatives from Detroit and surrounding areas, to focus climate adaptation efforts on priority areas, one of which is extreme heat events (Seroka, Kaiser, and Heany 2011). In the Michigan Climate and Health Adaptation Plan, state epidemiologists note that heat-related projections for Detroit are of particular concern, given that more frequent extreme heat events are projected to double or triple in number for Detroit, with temperatures exceeding $90^{\circ} \mathrm{F}$ approximately 30 to 50 days per year and exceeding $97^{\circ} \mathrm{F}$ approximately 25 to 50 days per year (Cameron et al. 2011).

\subsection{I-HEAT Feasibility Study}

In May 2012, the research team held a workshop with local decision makers to introduce and evaluate I-HEAT. This workshop was organized in collaboration with researchers from Michigan State University (MSU), the Michigan Department of Environmental Quality, and the Michigan Department of Community Health. The team was composed of environmental scientists, epidemiologists, behavioral scientists, and ecologists. Participants were selected through snowball sampling (Patton 2002), where members of this research network identified individuals to invite based on their current or potential role in local heat preparedness, and these individuals identified further contacts with relevant knowledge and experience. In particular, we identified and invited individuals who were most likely to make decisions about heat-related health interventions, representing local health, environmental planning and emergency preparedness agencies, departments, and organizations. Detroit's heat preparedness efforts, however, have not been extensive (White-Newsome et al. 2014). Thus, some participants were not identified as heat preparedness experts; rather they were identified as playing a role that could be relevant in future heatrelated climate change planning. Local decision makers from across mid-Michigan 
were invited, with an over-sampling of Detroit decision makers since the data in the I-HEAT prototype were Detroit-specific.

The workshop design was based largely on an active learning framework (Bens 2005) that emphasized participants' interactions with I-HEAT and other users. The day's agenda included icebreakers, large- and small-group activities, presentations, surveys, and focus groups, as well as morning refreshments and lunch. After an introductory welcome, a professional facilitator led a series of activities that required participants to get up and move around the room, physically indicating and discussing their knowledge, attitudes, and concerns about climate change and heat preparedness. This activity enabled participants to recognize their mental models (Morgan et al. 2002) about climate change, heat-related risks, and heat preparedness, which was especially important given the participants' large range of knowledge. Next, members of the research team gave a brief overview of heat vulnerability and epidemiology, followed by a description and demonstration of I-HEAT for the City of Detroit. After these presentations, participants formed teams of two or three and were provided prompts and scenarios (Table 1) to simulate real-world uses of I-HEAT and guide discussions. Participants interacted with the software online on laptop computers in groups and then completed individual surveys. After lunch, the research team divided the participants into three subgroups and conducted focus groups.

TABLE 1. Prompts used to guide discussion and assessment of I-HEAT for workshop attendees.

Prompt

1. Find a location where there is high risk during an extreme event. Are the areas near this location also at high risk? Repeat this for low risk areas.

2. Find a location with a high poverty rate. Does this location have a high or low risk?

3. Find a location that lacks vegetative cover. Does this location have a high or low risk?

4. Pick a location that is important to you. Does it have a high or low risk? Why do you think this is?

5. Explore another question that is important for you that you think I-HEAT might be able to address.

\section{Surveys}

Participants offered feedback after interacting with I-HEAT through a 12-question survey with seven quantitative, Likert-scale questions and five qualitative, openended questions. Questions pertained to terminology, opportunities for decision making, tool limitations, and satisfaction with various aspects of the tool. The surveys were piloted with two staff members from a local government agency outside of Southeast Michigan. Participants did not provide their contact information on the 
surveys, but they did note the sector they represented. This anonymity was intended to prevent social desirability bias (Crowne and Marlowe 1960), particularly since some participants knew members of the research team. Basic descriptive statistics were generated.

\section{Focus groups}

Participants were also divided into small groups to engage in a short set of semistructured, focus group questions at the end of the workshop. The multi-disciplinary research team developed questions (Table 2) that assessed I-HEAT's general strengths and weaknesses, potential applications, and features, such as terminology used (e.g., 'vulnerability' and 'at-risk') and definitions provided. Members of the research team facilitated the focus groups, which lasted approximately 1.5 hours and were audiotaped. A research team member with expertise in qualitative data analysis transcribed the audiotapes of the focus groups and conducted basic coding analysis (Patton 2002) by identifying themes and anomalous responses.

TABLE 2. Focus group questions answered by I-HEAT workshop attendees.

Question
1. Please describe one or two aspects of the I-HEAT software that you think are most useful to the work you do.
2. Are there any data missing from the I-HEAT application that you feel would help you with making decisions about
heat-related interventions? If so, please describe.
3. Please list any areas where the terminology used by I-HEAT software is different from what you use or are used to.
Are there other terms I-HEAT users may prefer or understand better?
4. Please provide one or two suggestions for how researchers could improve the I-HEAT software.
5. If you would consider using I-HEAT in your workplace, please provide a brief, specific example of how the software
may assist you and your colleagues in planning, implementing, or evaluating heat-related interventions.

\section{Results and Conclusions}

\subsection{I-HEAT Feasibility Study Findings}

Twenty-eight participants (including researchers) attended the workshop. Twentythree completed surveys and 26 participated in the focus group. Participants represented state agencies $(n=5)$, academic institutions $(n=11)$, non-profit organizations $(n=5)$, private research organizations $(n=2)$, local health departments $(n=$ $4)$ and the National Weather Service $(n=1)$. As summarized in Table 3, on average, participant satisfaction with I-HEAT fell closest to 'satisfied' regarding the types of 
data $($ mean $=3.86)$, user-friendliness of the web interface $($ mean $=3.64)$, general appearance of the web interface $($ mean $=4.09)$ and the graphics of the application (mean $=3.86)$. On average, participants were between 'unsatisfied' and 'neither satisfied or unsatisfied' with the performance, or speed, of the application. During the workshop we did encounter an unforeseen technical problem with the server on which I-HEAT is hosted, which limited the number of simultaneous users. Scores in the performance and speed category likely reflect this experience.

TABLE 3. Participant satisfaction with I-HEAT data, appearance, and performance.

\begin{tabular}{|c|c|c|c|c|c|c|c|c|c|c|}
\hline \multirow[t]{2}{*}{ Prompt } & \multicolumn{2}{|c|}{$\begin{array}{l}\text { Extremely } \\
\text { Unsatisfied }\end{array}$} & Uns & atisfied & $\begin{array}{r}\mathrm{N} \\
\text { Satis } \\
\text { Uns }\end{array}$ & $\begin{array}{l}\text { either } \\
\text { fied nor } \\
\text { atisfied }\end{array}$ & Sat & tisfied & \multicolumn{2}{|c|}{$\begin{array}{l}\text { Extremely } \\
\text { Satisfied }\end{array}$} \\
\hline & No. & $(\%)$ & No. & $(\%)$ & No. & $(\%)$ & No. & $(\%)$ & No. & $(\%)$ \\
\hline $\begin{array}{l}\text { The types of data (e.g., temperature, } \\
\text { vulnerability, demographics) decision makers } \\
\text { can select to view }(n=22)\end{array}$ & 0 & (0) & 0 & (0) & 6 & $(27.3)$ & 13 & (59.1) & 3 & (13.6) \\
\hline $\begin{array}{l}\text { The user-friendliness of the web interface } \\
(n=22) \\
(5=\text { easy to use } 1 \text { = too complicated })\end{array}$ & 0 & (0) & 4 & (18.2) & 4 & $(18.2)$ & 9 & $(40.9)$ & 5 & $(22.7)$ \\
\hline $\begin{array}{l}\text { The general appearance of the web interface } \\
(n=22)\end{array}$ & 0 & $(0)$ & 1 & (4.5) & 1 & $(4.5)$ & 15 & $(68.2)$ & 5 & $(22.7)$ \\
\hline $\begin{array}{l}\text { The graphics of the application } \\
\text { (e.g., map outputs) }(n=20)\end{array}$ & 0 & (0) & 1 & $(5.0)$ & 2 & $(10.0)$ & 14 & $(70.0)$ & 3 & $(15.0)$ \\
\hline $\begin{array}{l}\text { The performance (e.g., speed) of the } \\
\text { application }(n=23)\end{array}$ & 2 & $(8.7)$ & 11 & $(47.8)$ & 5 & $(21.7)$ & 3 & $(13.0)$ & 2 & (8.7) \\
\hline
\end{tabular}

\section{What Aspects of I-HEAT Are Most Useful for Local Decision Makers?}

Pilot users found I-HEAT to be a "quick" and "user-friendly" tool with a simple interface that made it "easy to see what's going on and help prioritize." Participants noted as useful three primary aspects of I-HEAT software: 1) localized analysis, 2) spatial visualization, and 3) integration of multiple types of data. According to many participants, a resource with this combination of characteristics is not currently "readily available," and I-HEAT would help planning, policy-making, identification of resources, and targeting of services for both climate mitigation (e.g., tree canopy density, green roof installation) and adaptation (e.g., cooling centers, health education, block group programs, transportation, emergency care) within jurisdictions. The software allowed them to understand "the where" of heat health risks and maintain a "strong sense of place," which they deemed to be key contributions of the software. Participants commented that they would be more likely to 
use tools such as I-HEAT with its localized data to determine where "predictors of heat-related health outcomes converge" than to use those providing smaller-scale, regional information. Participant comments included the following:

I think the vulnerability map is extremely useful, not only in relation to heat but for other public health issues. Being able to identify areas with high senior populations, for example, and show that visually could be very useful.

The spatial aspect of heat vulnerability is critical, and this is a unique tool to address that. It could help researchers/practitioners to localize areas of concern/ priority.

Spatial information is a critical part of the heat event impacts on human health; good to have a navigable tool that addresses this.

Specifically, participants emphasized that the amount and types of data were useful because they enabled a local understanding of relative risk and spatial variability among the variables. Participants also liked the inclusion of historical data, as well as local scale temperature and vegetative cover data. They commented that these data were more informative than the often-insufficient weather data frequently collected at airports, or the health data frequently collected at zip code or county levels. Finally, some participants noted that I-HEAT's variables effectively depicted cumulative and additive relationships of multiple risk factors.

\section{Improving I-HEAT}

Survey respondents had many useful suggestions for improving I-HEAT; most of these were related to the software's performance, its terminology, and the information it provides to users. The hands-on demonstration of I-HEAT involved small groups of workshop participants working through sample case studies. As mentioned above, some groups experienced limited performance due to inadequate bandwidth. Updates to the site bandwidth were implemented immediately following the workshop. Second, respondents requested that two layers in I-HEAT, Risk and Vulnerability, be distinctly differentiated. Vulnerability is the census tractspecific HVI value. Risk is a census tract-specific estimation of the contribution of the HVI value and satellite-derived land surface temperature (Conlon et al. 2014). Respondents noted that the interpretation of the term 'risk' can vary depending on the audience (e.g., community leaders, local health departments, policymakers). Similarly, the term 'vulnerability' is presented as both a layer and a tab within the 
tool. We addressed confusion about the difference between 'risk' and 'vulnerability' by providing detailed definitions within the tool for easy reference wherever the terms are present. Finally, survey respondents voiced concerns over the data used to create the tabs and layers within I-HEAT. For instance, participants were wary of the vulnerability tab, in which an HVI-composed of health, demographic, socioeconomic, and environmental variables - was mapped at the census tract level within the tool geography. We addressed this concern by providing improved descriptions of the data in the "About" tab in the I-HEAT software.

\section{How Might Local Decision Makers Use I-HEAT?}

As Table 4 shows, $65 \%$ of participants indicated that they were likely or extremely likely to use the software in their work, and $90 \%$ were likely or extremely likely to use I-HEAT if their suggestions were integrated into an updated version. To better understand the potential of I-HEAT, we also asked the participants to describe how they foresaw using the software in their work.

TABLE 4. Participant mean likelihood of using I-HEAT.

\begin{tabular}{llcccccccccc}
\hline & \multicolumn{2}{c}{$\begin{array}{c}\text { Extremely } \\
\text { Unlikely }\end{array}$} & \multicolumn{2}{c}{ Unlikely } & \multicolumn{2}{c}{ Not Sure } & & \multicolumn{2}{c}{ Likely } & \multicolumn{2}{c}{$\begin{array}{c}\text { Extremely } \\
\text { Likely }\end{array}$} \\
\cline { 2 - 11 } & No. & $(\%)$ & No. & $(\%)$ & No. & $(\%)$ & No. & $(\%)$ & No. & $(\%)$ \\
\hline $\begin{array}{l}\text { How likely are you to use I-HEAT, if it } \\
\text { were available to you? }(n=20)\end{array}$ & 0 & $(0)$ & 2 & $(10.0)$ & 5 & $(25.0)$ & 9 & $(45.0)$ & 4 & $(20.0)$ \\
$\begin{array}{l}\text { If your above suggestions were ad- } \\
\text { dressed, how likely are you to use }\end{array}$ & 0 & $(0)$ & 0 & $(0)$ & 2 & $(10.0)$ & 11 & $(55.0)$ & 7 & $(35.0)$ \\
$\begin{array}{l}\text { I-HEAT software, if it were available } \\
\text { for your region? }(n=20)\end{array}$ & & & & & & & & & & & \\
\hline
\end{tabular}

Respondents envisioned local health and planning departments using I-HEAT to identify areas of the city where, based on historical demographic and environmental data, vulnerable populations are likely to be found. By using this information prior to the spring and summer seasons when high temperatures can overwhelm public health and emergency response systems, the support systems in place can activate responses for the known vulnerable populations. Additionally, respondents discussed at length the potential utility of I-HEAT in developing adaptation activities (e.g., tree-planting, cooling center siting) during off-season times to ensure well-planned emergency responses. 
Although I-HEAT was presented within the city boundaries of Detroit, the pilot users saw potential in the tool's capacity to visualize heat vulnerability across the state, in an effort to plan for heat-health events in the context of an ever-changing (and hotter) Michigan climate.

\subsection{Strengths and Limitations}

This feasibility study could have been improved. The workshop was a day-long event, which may have deterred key decision makers from attending if they were unable to step away from the daily demands of their job for a full work day. As a remedy, researchers could have shortened the workshop or provided additional approaches for testing I-HEAT, such as a self-directed interaction and survey administered online. Furthermore, the sample size of 28 participants yielded informative results, but additional feasibility testing in other cities that have implemented climate adaptation and mitigation plans could be helpful to determine if 1) I-HEAT would be integrated into those efforts and 2) how similarly or differently end-users evaluate I-HEAT. Finally, the range of knowledge and the varied professional roles of survey respondents may complicate the interpretation of survey findings. For instance, participants who do not work directly on heat and health issues may not immediately see how the tool could assist them in their professional work, and they may respond to survey questions accordingly. We attempted to address this concern by providing a basic presentation on heat epidemiology to ensure that participants shared the same baseline knowledge.

Despite these limitations, the feasibility study advanced knowledge among the research team and participants. Specifically, survey and focus group data provided the research team with valuable information about the strengths and weaknesses of I-HEAT to inform next steps in tool development and implementation. The workshop provided new information to local decision makers, many of whom may not have had previous knowledge of heat epidemiology and vulnerability or potential heat preparedness interventions. Through the in-person workshop with diverse stakeholders, the feasibility study also enabled cross-sector, cross-disciplinary discussions that are vital to successful sustainability and climate planning initiatives.

\subsection{Future Directions for I-HEAT}

Future versions of I-HEAT will continue to build on the knowledge gained through collaboration with state and local experts on extreme heat and health, which may 
increase the generalizability of the tool. The research team is currently working to identify ways to expand I-HEAT with additional data that reflect community resources and health behaviors (e.g., transportation, social services, air conditioner usage), as these factors also contribute to vulnerability (Sampson et al. 2013). For example, after the workshop, cooling center locations were added to the web interface to help users see if and how current heat preparedness interventions aligned with the locations of vulnerable populations. In Detroit, the Detroit Climate Action Collaborative is now leading development of a Climate Action Plan. If updated, I-HEAT may assist in these efforts by helping decision makers target adaptation and mitigation strategies in ways that protect populations most vulnerable to local climate change. Most heat-related vulnerability studies are published in academic journals and are not usually tailored to communicate the results to an audience of practitioners. I-HEAT uniquely presents research-based information into a tool that can communicate complex environmental health relationships in an accessible manner. Workshop participants stressed that mapping tools like I-HEAT are relevant to audiences beyond environmental health practitioners. For instance, environmental planners, urban planners, and sustainability coordinators could use a tool like I-HEAT to identify fine-scale geographic areas where increased tree or vegetation coverage could result in co-benefits of greenhouse gas mitigation, stormwater management, and the reduction of the local UHI.

\subsection{Conclusions}

Increasing resiliency to climate impacts on human health requires integrated coordination among climate and health scientists, as well as municipal-level decision makers and planners. With the mass proliferation of climate adaptation tools (e.g., www.cakex.org), efforts to conduct feasibility studies of these tools are vital. As our study illustrates, end-users can offer insights that improve the tool's usability, clarify its language, expand its applicability, and contextualize its findings. The iterative development process of tools like I-HEAT provides benefits to producers and users alike. Actions to reduce exposure to extreme heat should consider practices that do not exacerbate the problem of climate change, but rather confer co-benefits to natural and human systems. This feasibility analysis suggests the possibility of developing decision-making tools that contribute to developing programs and interventions that support sustainable urban living. 


\section{Acknowledgements}

We thank collaborators at Michigan State University, the Michigan Department of Community Health, and the Michigan Department of Environmental Quality for co-coordinating pilot activities. We thank Susan Maxwell who provided technical support and guidance in this research project. Also, we greatly appreciate local decision makers who offered their time and feedback on I-HEAT. This research was supported by the National Aeronautics and Space Administration (NASA, NNH11CD21C), grant R-18-EH000348 from the U.S. Centers for Disease Control and Prevention, and grant R21 ES020156 from the National Institute for Environmental Health Sciences. The University of Michigan Risk Science Center also provided fellowship funding to support the feasibility study.

\section{References}

Anderson, G. Brooke, and Michelle L. Bell. 2011. "Heat Waves in the United States: Mortality Risk during Heat Waves and Effect Modification by Heat Wave Characteristics in 43 U.S. Communities." Environmental Health Perspectives 119 (2): 210-218. doi:10.1289/ehp.1002313.

Arnfield, A. John. 2003. "Two Decades of Urban Climate Research: A Review of Turbulence, Exchanges of Energy and Water, and the Urban Heat Island." International Journal of Climatology 23 (1): 1-26. doi:10.1002/joc.859.

Astrand, P. O., K. Rodahl, H. A. Dahl, and S. B. Stromme. 2003. Textbook of Work Physiology: Physiological Bases of Exercise (Fourth Edition). New York: McGraw-Hill.

Basu, R. 2002. "Relation between Elevated Ambient Temperature and Mortality: A Review of the Epidemiologic Evidence." Epidemiologic Reviews 24 (2): 190-202. doi:10.1093/epirev/mxf007.

Bens, I. 2005. "Understanding Participation." In Facilitating with Ease: Core Skills for Facilitators, Team Leaders and Members, Managers, Consultants, and Trainers. San Francisco: Jossey Bass.

Bhaduri, Budhendra, Edward Bright, Phillip Coleman, and Marie L. Urban. 2007. "LandScan USA: A High-resolution Geospatial and Temporal Modeling Approach for Population Distribution and Dynamics." GeoJournal 69 (1-2): 103-117. doi:10.1007/s10708-007-9105-9.

Bierbaum, Rosina, Joel B. Smith, Arthur Lee, Maria Blair, Lynne Carter, F. Stuart Chapin, III, Paul Fleming, Susan Ruffo, Missy Stults, Shannon McNeeley, Emily Wasley, and Laura Verduzco. 2013. "A Comprehensive Review of Climate Adaptation in the United States: More than Before, but Less than Needed." Mitigation and Adaptation Strategies for Global Change 18 (3): 361-406. doi:10.1007/s11027-012-9423-1.

Borden, Kevin, and Susan Cutter. 2008. "Spatial Patterns of Natural Hazards Mortality in the United States." International Journal of Health Geographics 7 (1): 64.

Cameron, L., M. Stanburry, R. Wahl, and S. Manente. 2011. Michigan Climate and Health Adaptation Plan: 2010-2015 Strategic Plan. Michigan Department of Community Health. http://www.michigan.gov/documents/mdch/MDCH_climate_change_strategicPlan_final_1-24-2011_343856_7.pdf. 
Conlon, Kathryn C., Natalie R. Sampson, Robert Rommel, Geoffrey Jacquez, and Marie S. O’Neill. 2014. "Internet-Based Heat Evaluation and Assessment Tool (I-HEAT): Development of a Novel Visualization and Decision-support Tool for Extreme Heat Preparedness in Detroit, Michigan.” Michigan Journal of Sustainability.

Crowne, D. P., and D. Marlowe. 1960. "A New Scale of Social Desirability Independent of Psychopathology." Journal of Consulting Psychology 24:349-354.

Curriero, Frank C., Karlyn S. Heiner, Jonathan M. Samet, Scott L. Zeger, Lisa Strug, and Jonathan A. Patz. 2002. "Temperature and Mortality in 11 Cities of the Eastern United States." American Journal of Epidemiology 155 (1): 80-7.

Frumkin, H. 2002. “Urban Sprawl and Public Health.” Public Health Reports 117:201-217.

Hess, Jeremy J., Paul J. Schramm, and George Luber. 2014. "Public Health and Climate Change Adaptation at the Federal Level: One Agency's Response to Executive Order 13514." American Journal of Public Health 104 (3): e22-e30. doi:10.2105/AJPH.2013.301796.

Imhoff, Marc L., Ping Zhang, Robert E. Wolfe, and Lahouari Bounoua. 2010. "Remote Sensing of the Urban Heat Island Effect across Biomes in the Continental USA." Remote Sensing of Environment 114 (3): 504-513. doi:10.1016/j.rse.2009.10.008.

IPCC (Intergovermental Panel on Climate Change). 2007. Climate Change 2007: The Physical Science Basis. Contribution of Working Group I to the Fourth Assessment Report of the Intergovernmental Panel on Climate Change, edited by S. Solomon, D. Qin, M. Manning, Z. Chen, M. Marquis, K. B. Averyt, M. Tignor and H. L. Miller. Cambridge, UK and New York: Cambridge University Press.

Johnson, Daniel P., Austin Stanforth, Vijay Lulla, and George Luber. 2012. "Developing an Applied Extreme Heat Vulnerability Index Utilizing Socioeconomic and Environmental Data." Applied Geography 35 (1): 23-31. doi:10.1016/j.apgeog.2012.04.006.

Loughnan, M. E., N. J. Tapper, T. Phan, K. Lynch, and J. A. McInnes. 2013. A Spatial Vulnerability Analysis of Urban Populations during Extreme Heat Events in Australian Capital Cities. Gold Coast: National Climate Change Adaptation Research Facility.

Meehl, Gerald A., and Claudia Tebaldi. 2004. "More Intense, More Frequent, and Longer Lasting Heat Waves in the 21st Century." Science 305 (5686): 994-997. doi:10.1126/science.1098704.

Miles, E. L., A. K. Snover, L. C. Whitely Binder, E. S. Sarachik, P. W. Mote, and N. Mantua. 2006. "An Approach to Designing a National Climate Service." Proceedings of the National Academy of Sciences 103 (52): 19616-19623. doi:10.1073/pnas.0609090103.

Morgan, M., B. Fischhoff, A. Bostrom, and C. Atman. 2002. Risk Communication: A Mental Models Approach. New York: Cambridge University Press.

Oleson, K. W., A. Monaghan, O. Wilhelmi, M. Barlage, N. Brunsell, J. Feddema, L. Hu, and D. F. Steinhoff. 2013. "Interactions between Urbanization, Heat Stress, and Climate Change." Climatic Change. doi:10.1007/s10584-013-0936-8.

O’Neill, Marie S., Dana K. Jackman, Michelle Wyman, Xico Manarolla, Carina J. Gronlund, Daniel G. Brown, Shannon J. Brines, Joel Schwartz, and Ana V. Diez-Roux. 2010. "US Local Action on Heat and Health: Are We Prepared for Climate Change?" International Journal of Public Health 55 (2): 105-112. doi:10.1007/s00038-009-0071-5.

Patton, M. 2002. Qualitative Research and Evaluation Methods (Third Edition). Thousand Oaks: Sage Publications, Inc.

Reid, Colleen E., Jennifer K. Mann, Ruth Alfasso, Paul B. English, Galatea C. King, Rebecca A. Lincoln, Helene G. Margolis, Dan J. Rubado, Joseph E. Sabato, Nancy L. West, Brian Woods, 
Kathleen M. Navarro, and John R. Balmes. 2012. "Evaluation of a Heat Vulnerability Index on Abnormally Hot Days: An Environmental Public Health Tracking Study." Environmental Health Perspectives 120 (5): 715-720. doi:10.1289/ehp.1103766.

Reid, Colleen E., Marie S. O’Neill, Carina J. Gronlund, Shannon J. Brines, Daniel G. Brown, Ana V. Diez-Roux, and Joel Schwartz. 2009. "Mapping Community Determinants of Heat Vulnerability.” Environmental Health Perspectives 117 (11): 1730-1736. doi:10.1289/ehp.0900683.

Sailor, D. J., and A. A. Pavlova. 2003. "Air Conditioning Market Saturation and Long-term Response of Residential Cooling Energy Demand to Climate Change." Energy 28 (9): 941-951. doi:10.1016/S0360-5442(03)00033-1.

Salamanca, F., M. Georgescu, A. Mahalov, M. Moustaoui, and M. Wang. 2014. "Anthropogenic Heating of the Urban Environment Due to Air Conditioning." Journal of Geophysical Research: Atmospheres 119 (10): 5949-5965. doi:10.1002/2013JD021225.

Sampson, Natalie R., Carina J. Gronlund, Miatta A. Buxton, Linda Catalano, Jalonne L. WhiteNewsome, Kathryn C. Conlon, Marie S. O’Neill, Sabrina McCormick, and Edith A. Parker. 2013. "Staying Cool in a Changing Climate: Reaching Vulnerable Populations during Heat Events." GlobalEnvironmental Change 23 (2): 475-484. doi:10.1016/j.gloenvcha.2012.12.011.

Seroka, C., P. Kaiser, and J. Heany. 2011. "Mapping Heat Vulnerability in Michigan.” In MPHI Annual Report. Michigan Public Health Institute.

Walsh, J. D., D. Wuebbles, K. Hayhoe, J. Kossin, K. E. Kunkel, G. Stephens, P. Thorne, R. Vose, M. F. Wehner, J. Willis, D. Anderson, V. Kharin, T. Knutson, F. Landerer, T. Lenton, J. Kennedy, and R. Somerville. 2014. "Appendix 3: Climate Science Supplement." In Climate Change Impacts in the United States: The Third National Climate Assessment, edited by J. M. Melillo, T. Richmond, and G. W. Yohe. US Global Change Research Program.

White-Newsome, Jalonne L., Sabrina McCormick, Natalie Sampson, Miatta A. Buxton, Marie S. O’Neill, Carina J. Gronlund, Linda Catalano, Kathryn C. Conlon, and Edith A. Parker. 2014. "Strategies to Reduce the Harmful Effects of Extreme Heat Events: A Four-City Study." International Journal of Environmental Research and Public Health 11 (2): 1960-1988.

Wilhelmi, Olga V., and Mary H. Hayden. 2010. "Connecting People and Place: A New Framework for Reducing Urban Vulnerability to Extreme Heat.” Environmental Research Letters, 5: 014021. doi:10.1088/1748-9326/5/1/014021

Wolf, Tanja, and Glenn McGregor. 2013. "The Development of a Heat Wave Vulnerability Index for London, United Kingdom.” Weather and Climate Extremes 1:59-68. doi:10.1016/j. wace.2013.07.004. 